\title{
MODEL FUNCTIONS WITH NEARLY PRESCRIBED MODULUS
}

\author{
YU. S. BELOV
}

\begin{abstract}
Let $\Theta$ be an inner function on the upper half-plane, and let $K_{\Theta}=$ $H^{2} \ominus \Theta H^{2}$ be the corresponding model subspace. A nonnegative measurable function $\omega$ is said to be strongly admissible for $K_{\Theta}$ if there exists a nonzero function $f \in K_{\Theta}$ with $|f| \asymp \omega$. Certain conditions sufficient for strong admissibility are given in the case where $\Theta$ is meromorphic.
\end{abstract}

\section{$\S 1$. INTRODUCTION}

Let $\Theta$ be an inner function on the upper half-plane $\mathbb{C}^{+}=\{z \in \mathbb{C}: \operatorname{Im} z>0\}$ (recall that a function on $\mathbb{C}^{+}$is said to be inner if it is analytic and bounded on $\mathbb{C}^{+}$and its angular boundary values are of modulus one a.e. on $\mathbb{R}$ ). The function $\Theta$ generates the so-called model subspace

$$
K_{\Theta}=H^{2} \ominus \Theta H^{2}
$$

of the Hardy space $H^{2}=H^{2}(\mathbb{R})=\left\{f \in L^{2}(\mathbb{R}): \hat{f}(\xi)=0\right.$ for a.e. $\left.\xi \in(-\infty, 0)\right\}$ (here $\hat{f}$ is the Fourier transform of $f$ ). These spaces play an important part in analysis and its applications to mathematical physics; we refer the reader to the monographs Cima, $\mathrm{N}, \mathrm{NF}$, and to the papers [Bl2, Bl3, $\mathrm{BH}, \mathrm{BBH}, \mathrm{D}, \mathrm{HM1}, \mathrm{HM}$, $\mathrm{MNH}$, which are particularly close to the subject matter of the present article (it is devoted to the moduli of model functions, i.e., of elements of $K_{\Theta}$ ). Here, the main attention is paid to the classical case where $\Theta(z)=e^{i \sigma z}, \sigma>0$; then $K_{\Theta}$ turns into $e^{i \sigma z / 2} P W_{\sigma / 2}$ (by $P W_{\sigma}$ we denote the Paley-Wiener space that consists of the entire functions of degree at most $\sigma$ and square integrable on $\mathbb{R}$ ).

As experience shows, the natural problem of describing the nonnegative functions $\omega$ that coincide a.e. on $\mathbb{R}$ with the modulus of a model function ( $\Theta$ being fixed) usually admits no satisfactory solution; see [D, HM1. Even the problem (much simpler at first glance) of finding a nonzero $f \in K_{\Theta}$ such that

$$
|f| \leq \omega \text { a.e. on } \mathbb{R}
$$

for a majorant $\omega: \mathbb{R} \mapsto[0,+\infty)$ fixed in advance, requires fairly deep analytic techniques. For example, the celebrated Beurling-Malliavin multiplier theorem is a result pertaining to the classical case of $\Theta(z)=e^{i \sigma z}, \sigma>0$.

Definition 1.1. A nonnegative function $\omega$ on $\mathbb{R}$ is said to be $\Theta$-admissible (in symbols: $\omega \in \operatorname{Adm}(\Theta))$ if there exists a nonzero function $f \in K_{\Theta}$ satisfying (1.1).

2000 Mathematics Subject Classification. Primary 30D50, 30D55.

Key words and phrases. Admissible function, Beurling-Malliavin theorem, model subspace, logarithmic integral.

Supported by RFBR, grant no. 06-01-00313. 
A necessary condition for $\Theta$-admissibility is the convergence of the logarithmic integral $\mathcal{L}(\omega)$

$$
\mathcal{L}(\omega)=\int_{\mathbb{R}} \Omega d \mathbf{P}, \quad \Omega=-\log \omega,
$$

where $d \mathbf{P}$ stands for the Poisson measure on $\mathbb{R}$ :

$$
d \mathbf{P}(x)=\frac{1}{\pi} \frac{d x}{1+x^{2}} .
$$

We assume that $\omega$ is Lebesgue measurable and $0 \leq \omega \leq 1$ a.e. on $\mathbb{R}$, so that the convergence of the integral $\mathcal{L}(\omega)$ means that $\mathcal{L}(\omega)>-\infty$. In should be noted that this condition never suffices for $\Theta$-admissibility (see $\underline{\mathrm{BH}}$ ). Sufficient conditions strongly depend on the structure of the generating function $\Theta$; they were studied in the papers [B12, Bl3, BH, BBH, HM1, HM2, MNH] already cited.

In the present paper, we consider a subset of $\operatorname{Adm}(\Omega)$ that consists of the so-called strongly admissible functions.

Definition 1.2. A function $\omega$ is said to be strongly admissible (more precisely, strongly $\Theta$-admissible; in symbols, $\omega \in \operatorname{sAdm}(\Theta)$ ) if there exists a function $f \in K_{\Theta}$ and two positive constants $C_{1}, C_{2}$ such that

$$
C_{1} \omega \leq|f| \leq C_{2} \omega \text { a.e. on } \mathbb{R}
$$

(for short, we often write $|f| \asymp \omega$ if such $C_{1}, C_{2}$ exist).

We need yet another notation:

$$
\operatorname{sADM}(\Theta)=\{-\log \omega, \omega \in \operatorname{sAdm}(\Theta)\} .
$$

Instead of the nonrealistic problem of finding $f \in K_{\Theta}$ with prescribed modulus (" $|f|=\omega$ a.e."), the function $f$ in (1.2) solves a weaker problem, specifically, that of finding a function in $K_{\Theta}$ with nearly prescribed modulus $(|f|$ is only comparable with $\omega$ on $\mathbb{R}:|f| \asymp \omega)$.

The main result of this paper (Theorem 2.4 in $\S 2$ ) describes a fairly wide subset of $\operatorname{sAdm}(\Theta)$ in case $\Theta$ is a meromorphic inner function. But we shall start the discussion with a partial and simpler case of that theorem pertaining to the classical situation of $\Theta(x)=e^{i \sigma x}, \sigma>0$ (Theorem 2.6). Before that, we need some auxiliary constructions.

The papers [B11, Bl2] show that conditions sufficient for admissibility are very often expressed in terms of the Hilbert transform $\widetilde{\Omega}$ rather than $\Omega$ itself or $\omega$. The Hilbert transform is understood in the following way:

$$
h(\Omega)(x)=\widetilde{\Omega}(x)=\frac{1}{\pi} \mathrm{P} . \mathrm{V} \cdot \int_{\mathbb{R}} \Omega(t)\left(\frac{1}{x-t}+\frac{t}{t^{2}+1}\right) d t, \quad x \in \mathbb{R} .
$$

The function $\widetilde{\Omega}$ is defined a.e. on $\mathbb{R}$ for every $\Omega \in L^{1}(\mathbf{P})$ (or, which is the same, for every $\omega$ with $\mathcal{L}(\omega)>-\infty)$. We also need the following version of the Hilbert transformation:

$$
h_{0}(\Omega)(x)=\frac{1}{\pi} \mathrm{P} \cdot \mathrm{V} \cdot \int_{\mathbb{R}} \frac{\Omega(t)}{x-t} d t .
$$

If $\Omega \in L^{1}(\mathbb{R})$, then $\widetilde{\Omega}=h_{0}(\Omega)+$ const.

A principal result in this paper (Theorem 2.6) is a "two-sided" refinement of the following statement due to Havin and Mashreghi and pertaining to $\operatorname{Adm}\left(e^{i \sigma z}\right)$ (rather than to $\left.\operatorname{sAdm}\left(e^{i \sigma z}\right)\right)$; see [HM2]. 
Theorem 1.3 (Havin-Mashreghi). Let $\Theta(z)=e^{i \sigma z}, \sigma>0$. Then any positive function $\omega$ with
a) $\left\|\widetilde{\Omega}^{\prime}\right\|_{\infty}<\frac{\sigma}{2}$
b) $\mathcal{L}(\omega)>-\infty$,

is $\Theta$-admissible.

Our Theorem 2.6 shows that a) and b) ensure the property $\omega \in \operatorname{sAdm}(\Theta)$ rather than the mere inclusion $\omega \in \operatorname{Adm}(\Theta)$. Strict admissibility in the classical case was studied in the unpublished paper $\mathrm{BB}$, the results of which cover Theorem 2.6 under the additional assumption $\Omega(x)=o(x),|x| \rightarrow+\infty$. In $[\mathrm{BB}$, the proof of Theorem 2.6 involves approximation of a subharmonic function by functions of the form $\log |g|$, where $g$ is an entire function. It seems that the way to (1.2) presented here is shorter. Moreover, our method applies to a much wider class of meromorphic functions $\Theta$. It should be noted that in $[\mathrm{BB}]$ it was proved that Theorem 2.6 is sharp. In that paper, for every $\sigma>0$ a function $\Omega$ was constructed such that $\Omega$ and $\widetilde{\Omega}$ are Lipschitz, $\sigma=\left\|\widetilde{\Omega^{\prime}}\right\|_{\infty}$, but $\Omega \notin \operatorname{sADM}\left(e^{i a z}\right)$ for every $a \in\left(0,2\|\widetilde{\Omega}\|_{\infty}\right)$ (however, $\Omega \in \operatorname{ADM}\left(e^{i a z}\right)$ for every $a>0$ ). Another proof of Theorem 2.6 is a consequence of the results of [LS] (that paper dealt with weighted Paley-Wiener spaces). I do not know if the methods of [LS] make it possible to cover other meromorphic functions $\Theta$, as in Theorem 2.4.

\section{§2. MAIN RESUlts}

We denote by $\left|K_{\Theta}\right|$ the set of all functions of the form $|f|$, where $f \in K_{\Theta}$. The following description of $\left|K_{\Theta}\right|$ was established in [HM1.

Theorem 2.1. Let $\Theta$ be an inner function, and let nonnegative functions $m$ and $\omega=e^{-\Omega}$ satisfy $\mathcal{L}(\omega)>-\infty, \mathcal{L}(m)>-\infty$, and $m \omega \in L^{2}(\mathbb{R})$. Then $m \omega \in\left|K_{\Theta}\right|$ if and only if there exists an inner function $I$ and an integer-valued function $n$ such that

$$
\arg \Theta+2 \widetilde{\Omega}=\widetilde{\log m}+2 \pi n+\arg I .
$$

Thus, if we solve equation (2.1) with the "unknowns" $m, n$, and $I$, and it turns out that $m \asymp 1$, a function $f \in K_{\Theta}$ with $|f| \asymp \omega$ will be found. It should be noted that $\inf m=0$ if $n$ is nonconstant. We will try to resolve equation (2.1) with $n=0$. Putting $\Phi=\arg \Theta+2 \widetilde{\Omega}$, we rewrite (2.1) in the form

$$
\Phi-\arg I=2 \widetilde{\log m} .
$$

So, if we find an inner function $I$ and a bounded function $m$ that is also bounded away from zero and satisfies $\widetilde{\log m}=\Phi-\arg I$, we will prove that $\omega$ is strongly admissible.

To state the main result of the paper, we need two definitions.

Definition 2.2. A partition of the real line into intervals $J_{k}=\left[d_{k}, d_{k+1}\right]$ (where $\left\{d_{k}\right\}$ is a strictly monotone increasing two-sided sequence) is said to be regular if

$$
\sup _{k \in \mathbb{Z}} \sum_{|k-l|>1} \frac{\left|J_{l}\right|^{2}}{\operatorname{dist}^{2}\left(J_{k}, J_{l}\right)}<+\infty .
$$

Here $\left|J_{k}\right|$ stands for the length of the interval $J_{k}$, and $\operatorname{dist}\left(J_{k}, J_{l}\right)$ is the distance between $J_{k}$ and $J_{l}$. For a regular partition, we have $\frac{\left|J_{k}\right|}{\left|J_{k+1}\right|} \asymp 1$. On the other hand, a partition is regular whenever $\left|J_{k}\right| \asymp 1$. It will be shown further that there exist regular partitions with inf $\left|J_{k}\right|=0$ (see Corollary 5.4).

Definition 2.3. A monotone increasing function $\Phi$ is said to be regular if there exists a sequence $\left\{d_{k}\right\}$ with $\Phi\left(d_{k}\right)=2 \pi k, k \in \mathbb{Z}$, such that the partition $J_{k}=\left[d_{k}, d_{k+1}\right]$ is regular and $\sup _{|\Phi(x)-\Phi(y)|<1} \frac{\Phi^{\prime}(x)}{\Phi^{\prime}(y)}<+\infty$. 
Now we are ready to state the main result.

Theorem 2.4. Suppose a meromorphic inner function $\Theta$ and a positive function $\omega=$ $e^{-\Omega} \in L^{2}(\mathbb{R})$ are such that
a) $\Omega \in L^{1}(\mathbf{P})$;
b) $\widetilde{\Omega} \in C^{2}(\mathbb{R})$;
c) the function $\arg \Theta+2 \widetilde{\Omega}$ is regular.

Then $\Omega \in \operatorname{sADM}(\Theta)$.

Before proving Theorem 2.4, we deduce from it two results pertaining to the classical case.

Theorem 2.5. Suppose a meromorphic inner function $\Theta$ and a positive function $\omega=$ $e^{-\Omega} \in L^{2}(\mathbb{R})$ satisfy
a) $\Omega \in L^{1}(\mathbf{P})$;
b) $\widetilde{\Omega} \in C^{1}(\mathbb{R})$;
c) $0<\inf _{\mathbb{R}}\left((\arg \Theta+2 \widetilde{\Omega})^{\prime}\right) \leq \sup _{\mathbb{R}}\left((\arg \Theta+2 \widetilde{\Omega})^{\prime}\right)<+\infty$.

Then $\Omega \in \operatorname{sADM}(\Theta)$.

Proof. The function $\arg \Theta+2 \widetilde{\Omega}$ is monotone increasing. There is a sequence $\left\{d_{k}\right\}$ with $(\arg \Theta+2 \widetilde{\Omega})\left(d_{k}\right)=2 \pi k, k \in \mathbb{Z}$; moreover, $d_{k+1}-d_{k} \asymp 1$. Consequently, the partition $\left\{J_{k}\right\}$, where $J_{k}=\left[d_{k}, d_{k+1}\right]$, is regular, and the function $\arg \Theta+2 \widetilde{\Omega}$ is also regular.

The following statement is an immediate consequence of Theorem 2.5.

Theorem 2.6. Let $\Theta(z)=e^{i \sigma z}$. If a function $\omega=e^{-\Omega} \in L^{2}(\mathbb{R})$ satisfies

a) $\Omega \in L^{1}(\mathbf{P})$, and

b) $\left\|\widetilde{\Omega^{\prime}}\right\|_{\infty}<\frac{\sigma}{2}$

then $\Omega \in \operatorname{sADM}(\Theta)$.

In some special cases, it turns out to be possible to get rid of the Hilbert transformation and to obtain sufficient conditions for strong admissibility in terms of the function $\Omega$ itself. For instance, as was shown in $\overline{\mathrm{BB}}$, if $\Omega \in C^{2}(\mathbb{R}) \cap L^{1}(\mathbf{P})$ monotonically increases on the ray $[A,+\infty)$ and monotonically decreases on $(-\infty, A]$, then $\Omega \in \operatorname{sADM}\left(e^{i \sigma z}\right)$ for every $\sigma>0$. The results of [B11, Theorem 9] show that if

$$
\Omega \in L^{1}(\mathbf{P}), \quad\left\|\Omega^{\prime}\right\|_{\infty}<+\infty, \quad \text { and } \quad \Delta_{2}(\Omega) \in L^{1}(\mathbf{P}),
$$

where

$$
\Delta_{2}(\Omega)(t)=\sup _{x \in \mathbb{R}}|\Omega(x+t)-2 \Omega(x)+\Omega(x-t)|,
$$

then $\left\|\widetilde{\Omega^{\prime}}\right\|_{\infty}<+\infty$. This implies the strong admissibility of $\omega$ for $K_{e^{i \sigma z}}$ with sufficiently large $\sigma$. Theorem 2.5 is applicable not only when $(\arg \Theta)^{\prime} \asymp 1$ (as in the classical case). For example, if $B$ is a Blaschke product with zeros $z_{k}=x_{k}+i y_{k}(k \in \mathbb{Z})$ and inf $y_{k}=0$, then there is some hope to improve the behavior of $\arg \Theta$ with the help of $2 \widetilde{\Omega}$. For instance, combining a result of [B13] (Corollary 6.1) and Theorem 2.5, we arrive at the following statement. Put

$$
A_{B}=\sum_{k \in \mathbb{Z}} \log \left(1+\frac{1-y_{k}^{2}}{\left(x-x_{k}\right)^{2}+y_{k}^{2}}\right) .
$$

Corollary 2.7. Let $B$ be a Blaschke product with zeros $z_{k}=x_{k}+i y_{k}, k \in \mathbb{Z}$. If a function $\omega=e^{-\Omega} \in L^{2}(\mathbb{R})$ is such that $\Omega$ belongs to $L^{1}(\mathbf{P})$ and is representable in the form $A_{B}+\Omega_{1}$, where $\widetilde{\Omega_{1}}$ is Lipschitz with a sufficiently small Lipschitz constant, then $\Omega \in \operatorname{sADM}(B)$. 


\section{§3. Outline of the Proof of Theorem 2.4: Preliminary Remarks}

In order to resolve equation (2.1), we formally rewrite it by using the Hilbert transformation. This suggests the following definition of $m$ :

$$
2 \log m=(\arg I-\Phi)^{\sim},
$$

where $I$ is an appropriate inner function that ensures the boundedness of $\log m$. (Strictly speaking, the definition of $\log m$ will not be so straightforward; here we only outline the principal idea.) The choice of $I$ is suggested by the rate of growth of $\Phi$ (see condition a) in Theorem 2.4). We define $I$ to be the Blaschke product with zeros $z_{k}=x_{k}+i y_{k}$, where a monotone increasing sequence $x_{k}$ behaves in the same way as the numbers $d_{k}$ determined by $\widetilde{\Phi}\left(d_{k}\right)=2 \pi k$. We must specify the choice of $x_{k}$ and $y_{k}$ so as to ensure the boundedness of the right-hand side of (2.2) on $\mathbb{R}$. Some preliminary remarks are required for that (recall that $h(f)$ and $\widetilde{f}$ denote the Hilbert transform with the regularized Cauchy kernel, and $h_{0}(f)$ is the usual Hilbert transform).

Lemma 3.1. Suppose $f \in L^{1}(a, b), f \equiv 0$ off $[a, b]$, and $\int_{\mathbb{R}} f(s) d s=0$. Then

$$
\left|h_{0}(f)(x)\right| \leq \frac{1}{\pi} \frac{\int_{a}^{b} \int_{a}^{t}|f(s)| d s d t}{\operatorname{dist}^{2}(x,[a, b])}, \quad x \notin[a, b] .
$$

Proof. Let $F$ be the primitive for $f$ with $F(a)=0$. Then $F(b)=0$. If $x \in[a, b]$, then

$$
\left|h_{0}(f)(x)\right|=\frac{1}{\pi}\left|\int_{a}^{b} \frac{f(t)}{x-t} d t\right|=\frac{1}{\pi}\left|\int_{a}^{b} F(t) \frac{d t}{(x-t)^{2}}\right| \leq \frac{1}{\pi} \cdot \frac{\int_{a}^{b}|F(t)| d t}{\operatorname{dist}^{2}(x,[a, b])} .
$$

Lemma 3.2. If $f$ is as in Lemma 3.1, then

$$
\left|h_{1}(f)(x)\right| \leq\left|h_{0}(f)(x)\right|+\int_{a}^{b} \frac{\left|\int_{a}^{t} f(s) d s\right|}{t^{2}+1} d t, \quad x \in \mathbb{R} .
$$

Proof. It is easily seen that

$$
\left|h(f)(x)-h_{0}(f)(x)\right|=\left|\int_{a}^{b} f(t) \frac{t}{t^{2}+1} d t\right|=\left|\int_{a}^{b} \int_{a}^{t} f(s) d s\left(\frac{t}{t^{2}+1}\right)^{\prime} d t\right|
$$

and $\left|\left(\frac{t}{t^{2}+1}\right)^{\prime}\right| \leq \frac{1}{t^{2}+1}$

Here and in what follows, we define $I$ to be a meromorphic Blaschke product with zeros $z_{k}=x_{k}+i y_{k} \quad\left(x_{k} \in \mathbb{R}, y_{k}>0, k \in \mathbb{Z}\right)$, where the sequence $\left\{x_{k}\right\}_{k \in \mathbb{Z}}$ is monotone increasing. It is known that

$$
(\arg I)^{\prime}(x)=\sum_{k} \frac{2 y_{k}}{\left(x-x_{k}\right)^{2}+y_{k}^{2}}, \quad x \in \mathbb{R}
$$

(see [HM2, p. 1259]). Under the condition

$$
\sum_{k \in \mathbb{Z}} \frac{y_{k}+y_{k}^{2}}{x_{k}^{2}}<+\infty
$$

we associate with $I$ a positive function $R_{I}$ on $\mathbb{R}$ defined by the formula

$$
R_{I}(x)=\sum_{k} \log \left(1+\frac{y_{k}^{2}}{\left(x-x_{k}\right)^{2}}\right), \quad x \in \mathbb{R} .
$$


Lemma 3.3. The function $R_{I}$ is integrable with respect to the Poisson measure (i.e., $\left.R_{I} \in L^{1}(\mathbf{P})\right)$ and

$$
-h\left(R_{I}\right)=\arg I-2 \pi n_{I}+\text { const, }
$$

where $n_{I}$ is the counting function for the function $x_{k}$, that is, $n_{I}(t)=\operatorname{card}\left\{k: 0 \leq x_{k}<t\right\}$ for $t \geq 0$, and $n_{I}(t)=-\operatorname{card}\left\{k: t<x_{k}<0\right\}$ for $t<0$.

Proof. The series (3.1) converges for $x \neq x_{k}$ because $\log \left(1+\frac{y_{k}^{2}}{\left(x-x_{k}\right)^{2}}\right) \leq \frac{y_{k}^{2}}{\left(x-x_{k}\right)^{2}}$ and the series $\sum_{k} \frac{y_{k}^{2}}{x_{k}^{2}}$ converges. In order to prove that $R_{I} \in L^{1}(\mathbf{P})$, we observe that

$$
\left.\pi \int_{\mathbb{R}} \log \left((x-X)^{2}+Y^{2}\right)\right) d \mathbf{P}(x)=\pi \log \left(X^{2}+(1+Y)^{2}\right), \quad Y \geq 0 .
$$

Indeed, let $Z:=X-i Y$. By the residue theorem,

$$
\begin{aligned}
\int_{\mathbb{R}} \frac{\log \left((x-X)^{2}+Y^{2}\right)}{1+x^{2}} d x & =2 \operatorname{Re} \int_{\mathbb{R}} \frac{\log (x-Z)}{1+x^{2}} d x \\
& =2 \operatorname{Re}\left[2 \pi \cdot i \operatorname{res}_{i}\left(\frac{\log (z-Z)}{1+z^{2}}\right)\right] \\
& =2 \operatorname{Re}[\pi i \log ((z-i) / i)]=2 \pi \log |X+i(Y+1)| \\
& =\pi \log \left(X^{2}+(1+Y)^{2}\right) .
\end{aligned}
$$

Letting $Y$ tend to zero, we see that the result remains true also for $Y=0$. Now, (3.3) with $X=x_{k}$ and $Y=y_{k}$ or $Y=0$ implies

$$
\int_{\mathbb{R}} \log \frac{\left(x-x_{k}\right)^{2}+y_{k}^{2}}{\left(x-x_{k}\right)^{2}} d \mathbf{P}(x)=\log \frac{x_{k}^{2}+\left(1+y_{k}\right)^{2}}{x_{k}^{2}+1} \leq \frac{2 y_{k}+y_{k}^{2}}{x_{k}^{2}+1} .
$$

Since the series $\sum_{k} \frac{y_{k}+y_{k}^{2}}{x_{k}^{2}}$ converges, we conclude that $R_{I} \in L^{1}(\mathbf{P})$.

Instead of (3.2), we verify the formula

$$
-h\left(R_{I}\right)+2 \pi n_{I}=\arg I+c .
$$

For this, we show that

a) the left-hand side of (3.4) can be defined at the points $x_{k}$ to become continuous;

b) off the $x_{k}$, the left-hand side is differentiable and its derivative coincides with $(\arg I)^{\prime}$, which implies (3.4) and (3.2).

a) The series defining $R_{I}$ converges in $L^{1}(\mathbf{P})$; consequently,

$$
h\left(R_{I}\right)(x)=\sum_{k \in \mathbb{Z}} h\left(\log \frac{\left(x-x_{k}\right)^{2}+y_{k}^{2}}{\left(x-x_{k}\right)^{2}}\right), \quad x \in \mathbb{R} .
$$

It is easily seen that the last series converges uniformly on every compact interval separated away from the $x_{k}$. We fix $l \in \mathbb{Z}$. On $\left(x_{l-1}, x_{l+1}\right)$, we have

$$
-h\left(R_{I}\right)=h\left(\log \left(x-x_{l}\right)^{2}\right)+r_{l},
$$

where the function $r_{l}$ is continuous on $\left(x_{l-1}, x_{l+1}\right)$. Note that

$$
h\left(2 \log \left|x-x_{l}\right|\right)=2 \arg \left(t-x_{l}\right)+\text { const }, \quad t \neq x_{l}
$$

( $\arg u:=0$ for $u>0$, and $\arg u:=\pi$ for $u<0$ ). From (3.5) and (3.6) we deduce that the function $-h\left(R_{I}\right)(t)$ has a first kind discontinuity (jumps down) when $t$ passes through $x_{l}$, whereas $2 \pi n_{I}$ jumps up (by $+2 \pi$ ). Consequently, the limits from the left and from the right of $-h\left(R_{I}\right)+2 \pi n_{I}$ at $x_{l}$ coincide.

b) Formal differentiation of the series defining $h\left(R_{I}\right)$ yields a series converging uniformly on every compact interval free of points $x_{k}$ (this is a direct consequence of the 
fact that the series defining $R_{I}$ converges in $L^{1}(\mathbf{P})$ ). Therefore, termwise differentiation on $\left(x_{l}, x_{l+1}\right)$, which annihilates $n_{I}$ on $\left(x_{l}, x_{l+1}\right)$, yields

$$
\begin{aligned}
\left(-h\left(R_{I}\right)\right. & \left.+2 \pi n_{I}\right)^{\prime}(x)=\sum_{k}-\left(h_{0}\left(\log \left(\left(x-x_{k}\right)^{2}+y_{k}^{2}\right)\right)-h_{0}\left(\log \left(x-x_{k}\right)^{2}\right)\right)^{\prime} \\
& =\sum_{k}-h_{0}\left(\left[\log \left(\left(x-x_{k}\right)^{2}+y_{k}^{2}\right)\right]^{\prime}\right)=-\sum_{k} h\left(\frac{2\left(x-x_{k}\right)^{2}}{\left(x-x_{k}^{2}\right)+y_{k}^{2}}\right) \\
& =\sum_{k} \frac{2 y_{k}}{\left(x-x_{k}\right)^{2}+y_{k}^{2}}=(\arg I)^{\prime}(x), \quad t \neq x_{k}, \quad k \in \mathbb{Z} .
\end{aligned}
$$

We observe that the function $h_{0}\left(\log \left(x-x_{k}\right)^{2}\right)$ is piecewise constant and $h_{0}\left(\frac{x}{1+x^{2}}\right)=$ $-\frac{1}{1+x^{2}}$.

\section{$\S 4$. Proof of Theorem 2.4}

By assumption, the function $\Phi=\arg \Theta+2 \widetilde{\Omega}$ is strictly monotone increasing on the real axis. Let $d_{k}$ satisfy $\Phi\left(d_{k}\right)=2 \pi k, k \in \mathbb{Z}$. We put $J_{k}=\left[d_{k}, d_{k+1}\right]$ and denote $a_{k}=\frac{1}{\left|J_{k}\right|} \int_{J_{k}} \Phi \in(2 \pi k, 2 \pi(k+1))$, where $a_{k}=2 \pi\left(k+q_{k}\right), q_{k} \in(0,1)$. Now we define $x_{k}=d_{k+1}-q_{k}\left|J_{k}\right|$, so that $x_{k}$ is an inner point of $J_{k}$. Let $n_{I}$ be the counting sequence for $\left\{x_{k}\right\}$, and $I$ the Blaschke product whose zeros are $x_{k}+i y_{k}$. There is no loss of generality in assuming that $d_{0}<0<x_{0}<d_{1}$ (consequently, $n_{I}\left(d_{k}\right)=k$ ). Put

$$
\Psi=\Phi-2 \pi n_{I} .
$$

Clearly,

$$
|\Psi| \leq 2 \pi, \quad \int_{J_{k}} \Psi=0 \quad \text { for every } \quad k \in \mathbb{Z}
$$

Indeed,

$$
\begin{aligned}
\int_{J_{k}} \Psi & =\left|J_{k}\right| a_{k}-2 \pi \int_{J_{k}} n_{I}\left|J_{k}\right| a_{k}-2 \pi k\left|J_{k}\right|+2 \pi\left(d_{k+1}-x_{k}\right) \\
& =2 \pi\left|J_{k}\right| q_{k}-2 \pi d_{k+1}+2 \pi x_{k}=0 .
\end{aligned}
$$

Note that $\Psi\left(d_{k}\right)=0$. Let

$$
2 \log m=R_{I}-\widetilde{\Psi}
$$

When the boundedness of $\log m$ is proved, the inclusion $\widetilde{\Psi} \in L^{1}(\mathbf{P})$ will follow from (4.1) because $R_{I} \in L^{1}(\mathbf{P})$ (see Lemma 3.3). Then we will be able to apply $h$ to the two sides of (4.1), obtaining

$$
\widetilde{2 \log m}=\widetilde{R_{I}}+\Psi+\text { const }=-\arg I+2 \pi n_{I}+\Phi-2 \pi n_{I}+\text { const },
$$

by Lemma 3.3. Since $\Phi=\arg \Theta+2 \widetilde{\Omega}$, it will follow that

$$
\widetilde{2 \log m}+\arg I=\arg \Theta+2 \widetilde{\Omega}+\text { const . }
$$

Since $m$ is bounded and separated away from zero, we conclude that $\omega$ is strongly admissible (see $\S 2$ ).

It remains to prove that $\log m$ is a bounded function. Fixing $k \in \mathbb{Z}$, we put

$$
J=J_{k-1} \cup J_{k} \cup J_{k+1} .
$$

Also, we define

$$
R_{I}^{s}(t)=\log \frac{1+\left(t-x_{s}\right)^{2}}{\left(t-x_{s}\right)^{2}}, \quad \Psi_{s}=\Psi \cdot \chi_{J_{s}}, \quad t \in \mathbb{R}, \quad s \in \mathbb{Z} .
$$


Now, formula (4.1) turns into

$$
\begin{aligned}
2 \log m= & \sum_{|s-k|>1} R_{I}^{s}-\sum_{|s-k|>1} h\left(\Psi_{s}\right) \\
& +\left\{\left[R_{I}^{k-1}+R_{I}^{k}+R_{I}^{k+1}\right]-h\left[\Psi_{k-1}+\Psi_{k}+\Psi_{k+1}\right]\right\} \\
= & V_{1}-V_{2}+V_{3} .
\end{aligned}
$$

We estimate $V_{1}$ :

$$
\begin{aligned}
0<\sum_{|s-k|>1} R_{I}^{s} & =\sum_{|k-s|>1} \log \left(1+\frac{y_{s}^{2}}{\left(x-x_{s}\right)^{2}}\right) \\
& \leq \sum_{|k-s|>1} \frac{y_{s}^{2}}{\left(x-x_{s}\right)^{2}} \leq \sum_{|k-s|>1} \frac{\left|J_{s}\right|^{2}}{\operatorname{dist}^{2}\left(J_{k}, J_{s}\right)}
\end{aligned}
$$

Since the partition $J_{k}$ is regular, the last sum is bounded uniformly in $k$. Now, we estimate $V_{2}$. Note that $\int_{J_{s}} \Psi_{s}=0$. Therefore, $\int_{J_{s}} \int_{J_{s}}|\Psi(s)| d s d t \leq 2 \pi\left|J_{s}\right|^{2}$. So, using Lemmas 3.1 and 3.2, we can estimate $|h(\Psi)|$ :

$$
\left|h\left(\Psi_{s}\right)(x)\right| \leq C_{1} \frac{\left|J_{s}\right|^{2}}{\operatorname{dist}^{2}\left(J_{k}, J_{s}\right)}+C_{2} \int_{J_{s}} \frac{d t}{t^{2}+1} .
$$

Summing over all $s$ with $|s-k|>1$ and using the fact that the partition $J_{k}$ is regular, we easily deduce that $V_{2}$ is uniformly bounded.

We pass to the subtlest estimate, namely, to that of $V_{3}$. Put

$$
\Upsilon=\Psi_{k-1}+\Psi_{k}+\Psi_{k+1}+2 \pi\left(\chi_{\left(x_{k-1}, d_{k+2}\right)}+\chi_{\left(x_{k}, d_{k+2}\right)}+\chi_{\left(x_{k+1}, d_{k+2}\right)}\right) .
$$

Thus,

$$
V_{3}=R_{I}^{k-1}+R_{I}^{k}+R_{I}^{k+1}+2 \pi h\left(\chi_{\left(x_{k-1}, d_{k+2}\right)}+\chi_{\left(x_{k}, d_{k+2}\right)}+\chi_{\left(x_{k+1}, d_{k+2}\right)}\right)-h(\Upsilon) .
$$

We show that the function $h(\Upsilon)$ is bounded. The function $\Upsilon$ itself is continuous and differentiable on $\left(d_{k-1}, d_{k+2}\right)$. Also, we emphasize that $\|\Upsilon\|_{\infty} \leq 6 \pi$ and $\left|\Upsilon^{\prime}(x)\right| \leq \frac{C}{\mid J_{k}}$, $x \in J$, where $C$ does not depend on $k$ because $\frac{1}{\left|J_{k}\right|}=\Upsilon^{\prime}(\xi)=\Phi^{\prime}(\xi)$ for $\xi \in J_{k}$. Put $m_{k}=\min \left\{\left|J_{k-1}\right|,\left|J_{k}\right|,\left|J_{k+1}\right|\right\}, M_{k}=\max \left\{\left|J_{k-1}\right|,\left|J_{k}\right|,\left|J_{k+1}\right|\right\}$. The partition $J_{k}$ is regular; consequently, $M_{k} \asymp m_{k}$. Furthermore,

$$
\begin{aligned}
h_{0}(\Upsilon)(x) & =\mathrm{P} . \mathrm{V} \cdot \int_{J} \frac{\Upsilon(t)}{x-t} d t \\
& =\mathrm{P} . \mathrm{V} \cdot \int_{|x-t| \leq m_{k}} \frac{\Upsilon(t)-\Upsilon(x)}{x-t} d t+\int_{|x-t|>m_{k}, x \in J} \frac{\Upsilon(t)}{x-t} d t .
\end{aligned}
$$

The absolute value of the first integral does not exceed $\frac{2 C m_{k}}{\left|J_{k}\right|}$, and the modulus of the second integral does not exceed $\|\Upsilon\|_{\infty} \cdot 2 \log \frac{2 M_{k}}{m_{k}}$. It can easily be shown that

$$
\left|h(\Upsilon)(x)-h_{0}(\Upsilon)(x)\right| \leq\|\Upsilon\|_{\infty} \cdot \int_{J} \frac{|t|}{t^{2}+1} d t
$$

(The last integral is bounded because the system $J_{k}$ is regular.) Consequently, the function $h(\Upsilon)$ is bounded on $J_{k}$ by a constant depending on $k$. It only remains to estimate the quantity

$$
R_{I}^{k-1}+R_{I}^{k}+R_{I}^{k+1}+2 \pi h\left(\chi_{\left(x_{k-1}, d_{k+2}\right)}+\chi_{\left(x_{k}, d_{k+2}\right)}+\chi_{\left(x_{k+1}, d_{k+2}\right)}\right) .
$$


We can use the operator $h_{0}$ in place of $h$ in this expression (because the difference is a bounded function), so that the expression in question turns into

$$
\begin{aligned}
{\left[\sum_{|s-k| \leq 1} \log \left(\left(x-x_{s}\right)^{2}+y_{s}^{2}\right)\right]-6 \log \left|x-d_{k+2}\right| } & \\
& =\sum_{|s-k| \leq 1}\left[\log \left(\left(x-x_{s}\right)^{2}+y_{s}^{2}\right)-2 \log \left|x-d_{k+2}\right|\right] .
\end{aligned}
$$

We treat the quantity $-6 \log \left|x-d_{k+2}\right|$ as the sum of three summands $-2 \log \left|x-d_{k+2}\right|$ and distribute them so as to compensate each of the terms $\log \left(\left(x-x_{s}\right)^{2}+y_{s}^{2}\right)$. Observe that

$$
\frac{\left|J_{s}\right|^{2}}{2 M_{k}^{2}} \leq \frac{\left(x-x_{s}\right)^{2}+y_{s}^{2}}{\left(x-d_{k+2}\right)^{2}} \leq \frac{4 M_{k}^{2}+\left|J_{s}\right|^{2}}{\left|J_{s}\right|^{2}}
$$

for $s=k-1, k, k+1$. Therefore, each summand in the last sum in (4.2) is bounded, and the theorem is proved.

\section{§5. Regularity condition: Examples of application of Theorem 2.4}

In this section, we discuss regularity conditions and show some applications of Theorem 2.4. First, we observe that regularity does not impose any growth restrictions on a function (in the sense that a regular function may grow arbitrarily fast). On the other hand, the regularity of the partition $J_{k}$ forbids slow growth (a regular function cannot grow more slowly than $\log ^{2}|x|$ at infinity). We recall that the regularity condition for a strictly monotone increasing function consists of two parts:

a) the regularity of the partition $J_{k}=\left[f^{-1}(2 \pi k), f^{-1}(2 \pi(k+1))\right]$;

b) $\sup _{|f(x)-f(y)|<1}\left|\frac{f^{\prime}(x)}{f^{\prime}(y)}\right|$ for all sufficiently large $x$ and $y$.

The following statement shows that sometimes b) is a consequence of a).

Lemma 5.1. If $f \in C^{2}(\mathbb{R})$ changes convexity to concavity and vice versa finitely many times, then b) follows from a).

Proof. There is no loss of generality in assuming that $f$ is concave (i.e., $f^{\prime \prime} \geq 0$ ). Let $x \in J_{k}$. We estimate $f^{\prime}(x)$ :

$$
f^{\prime}(y) \leq f^{\prime}(x) \leq f^{\prime}(z)
$$

for every $y \in J_{k-1}, z \in J_{k+1}$. Choose $y$ and $z$ in such a way that

$$
\begin{aligned}
f^{\prime}(y)\left(d_{k}-d_{k-1}\right) & =f^{\prime}(y)\left|J_{k-1}\right|=2 \pi, \\
f^{\prime}(z)\left(d_{k+2}-d_{k+1}\right) & =f^{\prime}(z)\left|J_{k+1}\right|=2 \pi .
\end{aligned}
$$

Since the partition $J_{k}$ is regular, we have $\left|J_{k}\right| \asymp\left|J_{k-1}\right| \asymp\left|J_{k+1}\right|$. Consequently, if $x \in J_{k}$, then $f^{\prime}(x) \asymp \frac{2 \pi}{\left|J_{k}\right|}$. Thus, b) is fulfilled.

For example, if $\Theta$ is the Blaschke product with zeros $i y_{k}, y_{k}>0$, then $\arg \Theta$ has one convexity-concavity change. Indeed,

$$
(\arg \Theta)^{\prime \prime}(x)=-2 x \sum_{k} \frac{y_{k}}{\left(x^{2}+y_{k}^{2}\right)^{2}} .
$$

In Theorem 2.4, it is assumed that the function $\arg \Theta+2 \widetilde{\Omega}$ is regular. As will be shown further, for many regular functions $\arg \Theta$ there are fairly many functions $\widetilde{\Omega}$ such that $\arg \Theta+2 \widetilde{\Omega}$ is also regular. 
Lemma 5.2. Suppose $f$ is a regular function with $f(0)=0$ and

$$
\sup _{1 / 2 \leq|x / y| \leq 2} \max \left(\frac{f(x)}{f(y)} ; \frac{f^{-1}(x)}{f^{-1}(y)}\right)<+\infty,
$$

and $g$ is a smooth function with $g(0)=0$ and $\left|g^{\prime}\right| \leq q f^{\prime}$ for some $q \in(0,1)$. Then $f+g$ is also regular.

Proof. It is easily seen that $|g| \leq q|f|$. Therefore, $f+g$ is monotone increasing and $f+g \asymp f$. Let $d_{k}$ and $d_{k}^{\prime}$ be determined by $f\left(d_{k}\right)=2 \pi k$ and $f\left(d_{k}^{\prime}\right)+g\left(d_{k}^{\prime}\right)=2 \pi k$. Next,

$$
2 \pi k=f\left(d_{k}^{\prime}\right)+g\left(d_{k}^{\prime}\right)=C f\left(d_{k}^{\prime}\right),
$$

where $C$ is bounded from above and from below by constants depending only on $g$. The properties of $f$ show that $d_{k}^{\prime} \asymp d_{k}$. Now, for some $x \in J_{k}=\left[d_{k}, d_{k+1}\right]$ we have

$$
f^{\prime}(x)\left(d_{k+1}-d_{k}\right)=2 \pi ;
$$

on the other hand, for some $y \in J_{k}^{\prime}=\left[d_{k}^{\prime}, d_{k+1}^{\prime}\right]$ we have

$$
\left(f^{\prime}(y)+g^{\prime}(y)\right)\left(d_{k+1}^{\prime}-d_{k}^{\prime}\right)=2 \pi=C f^{\prime}(y)\left(d_{k+1}^{\prime}-d_{k}^{\prime}\right) .
$$

Consequently, $\left|J_{k}\right|=d_{k+1}-d_{k} \asymp\left|J_{k}^{\prime}\right|=d_{k+1}^{\prime}-d_{k}^{\prime}$, and

$$
\operatorname{dist}\left(J_{k}, J_{l}\right) \asymp \operatorname{dist}\left(J_{k}^{\prime}, J_{l}^{\prime}\right) \text {. }
$$

Therefore, the partition $J_{k}^{\prime}$ is also regular; with it, $f+g$ is regular.

Lemma 5.2 allows us to give several applications of Theorem 2.4.

Corollary 5.3. Let functions $\Theta$ and $\omega$ satisfy $\arg \Theta+2 \widetilde{\Omega} \asymp(1+|x|)^{\beta}$ and $(\arg \Theta+2 \widetilde{\Omega})^{\prime} \asymp$ $(1+|x|)^{\beta-1}(1 \leq \beta<2)$. Then $\Omega \in \operatorname{sADM}(\Theta)$.

Proof. Consider the partition $J_{k}=\left[d_{k}, d_{k+1}\right]$. It is easily seen that

$$
\left|d_{k}\right| \asymp(1+|k|)^{\frac{1}{\beta}}, \quad\left|J_{k}\right| \asymp(1+|k|)^{\frac{1}{\beta}-1} .
$$

Then the function $\arg \Theta+2 \widetilde{\Omega}$ is regular. Indeed, take $s=(1 / \beta)-1 \in(-1 / 2,0)$. We know that $\left|J_{k}\right| \asymp|k|^{s} \leq 1$ and $\left|d_{k}\right| \asymp|k|^{s+1}$ for $k \neq 0$. Without loss of generality, we may assume that $0 \in J_{0}$. If $k>0$, then

$$
\sum_{|l-k|>1} \frac{\left|J_{l}\right|^{2}}{\operatorname{dist}^{2}\left(J_{k}, J_{l}\right)}=\sum_{l \leq 0}+\sum_{0<l<k / 2}+\sum_{k / 2 \leq l<k-1}+\sum_{l>k+1}=S_{1}+S_{2}+S_{3}+S_{4}
$$

First, we observe that $S_{1} \leq$ const $\sum_{l \leq 0}\left(d_{l}\right)^{-2}$, and the last sum is bounded uniformly in $k$. Second, if $l>k>0$, we have $\operatorname{dist}\left(J_{l}, J_{k}\right) \geq$ const $\left|J_{l}\right| \cdot|l-(k+1)|$. Consequently,

$$
S_{4} \leq \mathrm{const} \sum_{l>k+1} \frac{1}{|l-(k+1)|^{2}} .
$$

We note that for $0 \leq l<k-1$ we have

$$
\operatorname{dist}\left(J_{k}, J_{l}\right)=\sum_{n=l+1}^{k-1}\left|J_{n}\right| \asymp \sum_{n=l+1}^{k-1}|n|^{s} \asymp|k|^{s+1}-|l|^{s+1} .
$$


Therefore,

$$
\begin{aligned}
S_{2} & \leq \mathrm{const} \sum_{0<l<k / 2}\left(\frac{|k|^{s}}{|k|^{s+1}-|l|^{s+1}}\right)^{2} \\
& \leq \mathrm{const} \sum_{0<l<k / 2} \frac{1}{|k|^{2(s+1)}} \leq \mathrm{const}|k|^{-2 s-1} \leq \mathrm{const}, \\
S_{3} & \leq \mathrm{const} \sum_{k / 2 \leq l<k-1}\left(\frac{|k|^{s}}{|k|^{s+1}-|l|^{s+1}}\right)^{2} \leq \mathrm{const} \sum_{l<k-1} \frac{1}{(k-l)^{2}} .
\end{aligned}
$$

So, we have estimated the quantities $S_{1}, S_{2}, S_{3}$, and $S_{4}$ on the right of (5.1). For $k<0$ the proof is similar.

The above assumption allows us to prove that certain specific majorants are admissible. Here is an example.

Corollary 5.4. Let $B_{\alpha}$ denote the Blaschke product with the zeros $z_{k}=\operatorname{sgn}(k)|k|^{\alpha}+i$, $k \in \mathbb{Z}, 1 / 2<\alpha<1$. If a function $\omega=e^{-\Omega} \in L^{2}(\mathbb{R})$ satisfies

a) $\Omega \in L^{1}(\mathbf{P})$, and

b) $\left|\widetilde{\Omega}^{\prime}(x)\right|<C|x|^{1 / \alpha-1}$ for some $C<\frac{\pi}{\alpha}$, then $\Omega \in \operatorname{sADM}\left(B_{\alpha}\right)$.

Proof. This is a direct consequence of Corollary 5.3 and the estimate $\left(\arg B_{\alpha}\right)^{\prime}(x)=$ $\frac{2 \pi}{\alpha}|x|^{1 / \alpha-1}+O(1)$ (see [HM2, p. 1298]).

I am grateful to V. P. Havin for supervision and for useful advice during the preparation of this publication, and to A. D. Baranov for a discussion of the first version of Theorem 2.4, which made it possible to lift some redundant conditions imposed on $\omega$.

\section{REFERENCES}

[BB] A. D. Baranov and A. A. Borichev, Entire functions of exponential type with prescribed modulus on the real axis (unpublished).

[B11] Yu. S. Belov and V. P. Khavin, On a theorem of I. I. Privalov on the Hilbert transform of Lipschitz functions, Mat. Fiz. Anal. Geom. 11 (2004), no. 4, 380-407. (Russian) MR2114001 (2005k:26006)

[B12] Yu. S. Belov, Admissibility criteria for model subspaces with fast growth of the argument of the generating inner function, Zap. Nauchn. Sem. S.-Peterburg. Otdel. Mat. Inst. Steklov. (POMI) 345 (2007), 55-84; English transl. in J. Math. Sci. (New York). MR 2432176

[B13] N Necessary conditions of admissibility for some model subspaces, Algebra i Analiz (to appear).

[BH] A. D. Baranov and V. P. Khavin, Admissible majorants for model subspaces and arguments of inner functions, Funktsional. Anal. i Prilozhen. 40 (2006), no. 4, 3-21; English transl., Funct. Anal. Appl. 40 (2006), no. 4, 249-263. MR2307699 (2008c:30058)

[BBH] A. D. Baranov, A. A. Borichev, and V. P. Havin, Majorants of meromorphic functions with fixed poles, Indiana Univ. Math. J. 56 (2007), 1595-1628. MR2354693 (2008i:30034)

[Cima] J. A. Cima and W. T. Ross, The backward shift on the Hardy space, Math. Surveys Monogr., vol. 79, Amer. Math. Soc., Providence, RI, 2000. MR.1761913 (2002f:47068)

[D] K. M. D'yakonov, Moduli and arguments of analytic functions from subspaces in $H^{p}$ that are invariant under the backward shift operator, Sibirsk. Mat. Zh. 31 (1990), no. 6, 64-79; English transl., Siberian Math. J. 31 (1990), no. 6, 926-939 (1991). MR1097956 (92f:30049)

[dB] L. de Branges, Hilbert spaces of entire functions, Prentice-Hall, Englewood Cliffs, NJ, 1968. MR0229011 (37:4590)

[HM1] V. P. Havin and J. Mashreghi, Admissible majorants for model subspaces of $H^{2}$. I. Slow winding of the generating inner function, Canad. J. Math. 55 (2003), 1231-1263. MR2016246 (2004i:30029a)

[HM2] Admissible majorants for model subspaces of $H^{2}$. II. Fast winding of the generating inner function, Canad. J. Math. 55 (2003), 1264-1301. MR2016247 (2004i:30029b) 
[LS] Yu. I. Lyubarskii and K. Seip, Weighted Paley-Wiener spaces, J. Amer. Math. Soc. 15 (2002), no. 4, 979-1006. MR1915824 (2003m:46039)

$[\mathrm{MNH}]$ J. Mashreghi, F. L. Nazarov, and V. P. Havin, Beurling-Malliavin multiplier theorem: the seventh proof, Algebra i Analiz 17 (2005), no. 5, 3-68; English transl., St. Petersburg Math. J. 17 (2006), no. 5, 699-744. MR2241422 (2007g:42028)

[N] N. K. Nikol'skiū, Treatise on the shift operator. Spectral function theory, "Nauka", Moscow, 1980; English transl., Grundlehren Math. Wiss., Bd. 273, Springer-Verlag, Berlin, 1986. MR0575166 (82i:47013) MR0827223 (87i:47042)

[NF] B. Sz.-Nagy and C. Foiaş, Harmonic analysis of operators on Hilbert space, Amer. Elsevier Publ. Co., Inc., New York; Akad. Kiadó, Budapest, 1970. MR0275190 (43:947)

Department of Mathematics and Mechanics, St. Petersburg State University, Petrodvorets, Universitetskil Prospekt 20, St. Petersburg 198504, Russia

E-mail address: j_b_juri_belov@mail.ru

Received 20/DEC/2007

Translated by S. V. KISLYAKOV 